\title{
El saber de la experiencia del profesorado en la formación inicial: Reflexiones desde una asignatura de educación inclusiva
}

\author{
Teacher's Knowledge Experience in Initial Training: Reflections From an Inclusive \\ Education Course
}

A sabedoria da experiência do corpo docente em formação inicial: reflexões a partir de uma disciplina de educação inclusiva

Valeska Cabrera-Cuadros

Universidad de Barcelona

Barcelona, España

vcabrera@ub.edu

iD https://orcid.org/0000-0002-2560-6303

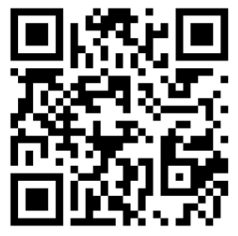

Claudia Soto-García

Universidad de Barcelona

Barcelona, España

claudiasotogarcia@gmail.com

https://orcid.org/0000-0003-1468-9235

Asunción López-Carretero

Universidad de Barcelona

Barcelona, España

asuncion.lopez@ub.edu

iD https://orcid.org/0000-0002-2548-4908

Recibido • Received • Recebido: 17 / 09 / 2019

Corregido • Revised • Revisado: 22 / 05 / 2021

Aceptado • Accepted • Aprovado: 14 / 06 / 2021

\begin{abstract}
Resumen: Poner el foco en la formación docente para proyectar una escuela inclusiva es clave. El objetivo de la presente investigación es reflexionar sobre la incorporación del saber de la experiencia en la formación inicial del profesorado, usando como fuente el relato de una profesora que implementa una propuesta didáctica inclusiva. Se realizó un seguimiento durante un semestre en la asignatura Teoría y práctica de la escuela inclusiva, donde participaron 43 estudiantes de segundo año del Grado de Educación Infantil en la Universidad de Barcelona. La investigación es de tipo cualitativa con un enfoque de indagación narrativa, donde se diseñaron 4 fases para llegar al texto de investigación final; fase 1, registro de textos de campo; fase 2, elaboración de textos intermedios; fase 3, regreso al campo con el texto intermedio para nuevas reflexiones; fase 4, elaboración de los textos de investigación final que conforman este estudio. Los resultados muestran que el estudiantado se va aproximando a proyectos inclusivos desde una dimensión personal al interaccionar con saberes que parten de la
\end{abstract}


http://doi.org/10.15359/ree.25-3.8

http://www.una.ac.cr/educare

educare@una.ac.cr

experiencia y del autoconocimiento. Por otro lado, surgieron algunas tensiones: dificultad para actuar frente a las libertades otorgadas en la clase; inicialmente dificultades para compartir sus experiencias de manera oral y escrita, y necesidad de respuestas estandarizadas. El estudio permitió analizar una práctica educativa que se va forjando en la construcción de espacios de convivencia, la cual invita a nuevas reflexiones en torno al saber de la experiencia para pensar en la escuela inclusiva.

Palabras claves: Experiencia; formación inicial docente; educación inclusiva; narrativa.

Abstract: Focusing on teachers' training is the key to project an inclusive school. This research aims to reflect on the incorporation of knowledge from experience in the initial teacher training, using the history of a teacher as a source that implements an inclusive didactic proposal. Monitoring was carried out during a semester in the course Theory and Practice of Inclusive Education from an Early Childhood Education degree at the University of Barcelona, in which 43 second-year students participated. This is a qualitative research design with a narrative inquiry approach, where 4 phases were designed to arrive at the final research text; phase 1, registration of fi eld texts; phase 2, preparation of intermediate texts; phase 3, return to the field with the intermediate text for further reflections; phase 4 , preparation of the final research texts that make up this study. The results s how that students approach inclusive projects from a personal dimension when interacting with the knowledge that comes from experience and self-knowledge. On the other hand, some tension arose: difficulty dealing with the freedom granted in class; initially, difficulties sharing their experiences orally and in writing, looking for standardized answers. This study allowed us to analyze an educational practice that is being forged in the construction of spaces for coexistence, which invites new reflections on the knowledge of the experience thinking about the inclusive school.

Keywords: Experience; initial teacher training; inclusive education; narrative.

Resumo: Concentrar-se na formação do corpo docente para projetar uma escola inclusiva é fundamental. O objetivo desta pesquisa é refletir sobre a incorporação de saberes oriundos da experiência na formação inicial de professores, tendo como fonte a história de uma professora que implementa uma proposta didática inclusiva. Foi realizado um acompanhamento durante um semestre na disciplina Teoria e prática da escola inclusiva, na qual participaram 43 estudantes do segundo ano da licenciatura em Educação Infantil na Universidade de Barcelona. A pesquisa é qualitativa com abordagem de investigação narrativa, com 4 fases para chegar ao texto final da pesquisa; fase 1, registro dos textos de campo; fase 2, preparação de textos intermediários; fase 3, retorno ao campo com o texto intermediário para novas reflexões e fase 4, elaboração dos textos finais de pesquisa que compõem este estudo. Os resultados mostram que o corpo docente está abordando projetos inclusivos em uma dimensão pessoal, interagindo com conhecimentos advindos da experiência e do autoconhecimento. Por outro lado, surgiram algumas tensões: dificuldade em atuar diante das liberdades conferidas na aula; dificuldades iniciais em compartilhar suas experiências oralmente e por escrito; e a necessidade de respostas padronizadas. $O$ estudo permitiu analisar uma prática educativa que se vai forjando na construção de espaços de convivência, que convida a novas reflexões sobre o conhecimento da experiência pensando a escola inclusiva.

Palavras-chave: Experiência; formação inicial de professores; educação inclusiva; narrativa. 


\section{Introducción}

Es indiscutible la importancia de incorporar el sentido de la inclusión en el currículo de formación inicial docente (FID). Al realizar una revisión bibliográfica sobre el tema nos percatamos de que algunos trabajos analizan los programas de estudio y evalúan estrategias metodológicas más activas y participativas (Arostegui Barandica y Darretxe Urrutxi, 2016; de la Rosa Moreno, 2017; García García y Benitez Gavira, 2014; Mendonça et al., 2015; Simón et al., 2018), donde se evidencia la necesidad de generar nuevas propuestas en las aulas universitarias para trabajar aspectos teóricos de la inclusión y para conocer estrategias didácticas que se estén llevando a cabo.

En la formación inicial del profesorado es fundamental la relación entre universidad y escuela (Hermida Vázquez et al., 2015) pues la mirada de la escuela y la mirada con la que se plantea la formación determina la dinámica entre esos dos contextos que se interrelacionan. Esto nos llevó a preguntarnos de qué manera se proyecta la escuela inclusiva en la universidad. Esta problemática emerge de un proyecto de investigación del grupo Experiencia, Saber y Formación de Educadoras y Educadores, Investigación y Acción (ESFERA) de la Universidad de Barcelona. ${ }^{1}$

Se presenta en este estudio, por medio de relatos de experiencia, una propuesta didáctica, donde una docente universitaria promueve un espacio educativo que releva el compartir experiencias y saberes en coherencia con los contextos de formación, incorporando una perspectiva que parte del reconocimiento personal y de las historias de quienes se están formando para ser maestros y maestras, capaces de sentir y crear contextos más inclusivos. Todo esto se desarrolla en una asignatura denominada Teoría y práctica de la escuela inclusiva que se imparte en el grado de Educación Infantil en la Universidad de Barcelona.

En la actualidad, hay propuestas y experiencias que abordan los desafíos para hacer que escuelas y aulas sean más inclusivas (Ainscow et al., 2012), es decir, hay una intención de acercamiento a contextos en continuo desarrollo, capaces de poner al alcance de todas las personas, diferentes oportunidades para la integración, la participación y el aprendizaje (Ainscow, 2001).

Existen estudios (Martínez Tornay, 2014; Rivera Iribarren, 2017; Rojas Cely y Quintero Peñaranda, 2013) que reconocen la responsabilidad de contribuir a la formación del futuro personal docente para que sea capaz de asumir los propios retos de la educación inclusiva y, en consecuencia, asumir las diversidades de las aulas e instaurar acciones pedagógicas incluyentes, apropiadas y pertinentes. No obstante, falta pensar en experiencias inclusivas (o no) que han vivido quienes se están formando para ser maestros y maestras que en un futuro acompañarán

\footnotetext{
${ }^{1}$ Proyecto de investigación titulado Relaciones educativas y creación del currículo: Entre la experiencia escolar y la formación inicial del profesorado. Indagaciones narrativas, EDU2016-77576-P.
} 
http://doi.org/10.15359/ree.25-3.8

http://www.una.ac.cr/educare

educare@una.ac.cr

la creación de nuevos proyectos en las escuelas. Poder rescatar y encarnarse de las historias que surgen en las aulas universitarias podría servir para visualizar ese gran objetivo de un mundo educativo no excluyente. En ello se focaliza la profesora a la que acompañamos en la asignatura; sus clases plantean una manera de estar presente con los saberes, con las historias y con las experiencias de cada estudiante.

El objetivo del presente estudio es reflexionar sobre la incorporación del saber de la experiencia en la FID, usando como fuente el relato de una profesora que implementa una propuesta didáctica inclusiva. En este sentido, hemos puesto la mirada en las posibilidades y dificultades que tiene el hecho de reconstruir significados del estudiantado a la hora de hablar de educación inclusiva, haciendo una conexión entre escuela y universidad, al pensarse como futuros maestros y maestras que miran su quehacer desde un prisma inclusivo.

Al hablar del saber de la experiencia a lo largo del estudio, lo entendemos como lo plantea Contreras Domingo (2013, pp. 129-130):

El acontecimiento novedoso que requiere ser pensado para preguntarse por su sentido ... como aquello que nos ocurre, que nos deja huella, que tiene un efecto personal. ... Es una expresión para nombrar ese saber sabio que ayuda a estar en el mundo con mayor amplitud y sensibilidad.

La experiencia se ubica dentro de lo que se piensa, se hace y se vive en una relación y del sentido que se le otorga a ello (Caine et al., 2019). Por tanto, la visión narrativa de la experiencia que hemos querido poner en juego atiende a una tridimensionalidad: espacio, tiempo y socialidad (Caine et al., 2013).

De manera más específica, ponemos atención en una práctica pedagógica en la universidad donde se valoran las interacciones con la diferencia y la alteridad, con la intención de integrar saberes que conecten mejor con el sentido y la experiencia del oficio docente desde una mirada inclusiva. En suma, exploramos un proceso de formación que nos permite replantear formas de acercarnos a la escuela desde la experiencia de las personas.

Desde este posicionamiento, queremos compartir lo vivido en la asignatura citada, que abrió la posibilidad para que el estudiantado pudiera pensarse desde sus propias historias de inclusión/exclusión escolares y personales y aproximarse a la toma de conciencia respecto al proyecto de escuela de la que quiere ser parte.

Como docentes nos preocupan las prácticas que se llevan a cabo en las aulas de FID y nos preguntamos cómo se están trabajando temas fundamentales como la educación inclusiva. Algunas de las cuestiones que inspiraron el estudio son: el incorporar el sentido personal del estudiantado ¿permite cambiar la perspectiva de una escuela inclusiva que pretende relevar 
http://doi.org/10.15359/ree.25-3.8

http://www.una.ac.cr/educare educare@una.ac.cr

el saber de la experiencia? ¿Qué posibilidades y dificultades surgen al incorporar el saber de la experiencia en la formación inicial? Entonces, ¿qué saberes reclama la FID para tratar temas relacionados a la escuela inclusiva?

\section{Marco teórico}

La perspectiva y la manera en que se enseña a los futuros cuerpos docentes es fundamental en la mejora de la escuela y en su planteamiento desde un sentido inclusivo (Durán Gisbert y Giné Giné, 2017). Ello ha demandado pensar en estrategias con principios pedagógicos que reflejen el respeto por el otro ser y donde haya un reconocimiento de la propia persona que está en las aulas formándose para ser docente (Alliaud y Suárez, 2011; Arbiol i González et al., 2016).

Es necesario que desde la FID se rediseñen estrategias de aprendizaje para documentar, describir y compartir experiencias (Marcelo et al., 2014). Sobre todo, cuando se necesita que la universidad y la escuela tengan lineamientos que confluyan, rompiendo la brecha entre estos dos espacios que, en ocasiones, suelen tener ciertos distanciamientos (Ramos Torres y Galindo Rodríguez, 2018). Desde el posicionamiento de este trabajo, la FID convive con la realidad contextual de la escuela.

Por otra parte, el tema de la educación inclusiva se ha convertido en una necesidad para repensar el rol y la actitud de los docentes y las docentes. El quehacer profesional supone nuevas condiciones para caminar hacia centros con una orientación inclusiva (Moriña Díez y Parrilla Latas, 2006).

Con la Declaración de Salamanca en 1994 se comenzó a tener presente en los programas de FID el tema de la educación inclusiva para impulsar cambios en los sistemas educativos (Duk et al., 2019) y así proyectar estrategias que respondiesen a los cambios de perspectivas que se comenzaba a vivir en las escuelas.

Es importante reflexionar respecto a qué prácticas didácticas se llevan a cabo en las aulas para tratar este tema, pues las decisiones pedagógicas podrían ayudar a generar en el estudiantado universitario un saber propio a partir de los planteamientos teóricos. Una alternativa para tal intención en la FID es incorporar la mirada que valora la experiencia (VélezCalvo et al., 2016). Específicamente en las asignaturas destinadas a la formación se reconoce una capacidad y actitud que beneficia la diversidad, fundamentada en el análisis crítico de la función de la escuela (Huguet i Comelles, 2006). De ahí emanan algunas preguntas claves: ¿Cómo se mira la escuela desde la universidad? ¿Qué prácticas se llevan a cabo en las aulas universitarias para pensar en la diversidad de las escuelas? ¿Se reflexiona sobre el lugar propio que se ocupa mientras se viven procesos de formación?

Para tejer prácticas inclusivas y conseguir un espacio social en la universidad, específicamente en la FID, es necesaria una educación dialógica y una educación que ponga 
http://doi.org/10.15359/ree.25-3.8

http://www.una.ac.cr/educare

educare@una.ac.cr

atención a lo que nos pasa, que esté abierta a pensar en la relación con las otras personas (Porta et al., 2015). Es decir, tenemos que repensar las prácticas en los espacios educativos para convertirlas en un punto de encuentro. Aquí tomamos como eje el saber personal y aquel que se construye colectivamente. Se trata de entender la formación como un proceso constructivo con sentido y significado a partir de la experiencia personal y profesional, el cual tiene gran valor al ser considerado en las aulas de FID. Por lo tanto, para encaminarnos hacia la inclusión debemos mirar las prácticas educativas que llevamos al aula donde se instruye el profesorado (Pérez Santalla, 2017) y en una dimensión que nos inspira a cuidar y cultivar las relaciones humanas (Arbiol i González et al., 2016).

El oficio docente, considerado un oficio de lo humano, requiere de la elaboración de una serie de saberes (Blanco García et al., 2015) pues, como dice Contreras Domingo (2013): el saber docente va más allá de un saber técnico y disciplinar, es más bien un saber ligado a la vida y a la propia experiencia. En relación con esto, entendemos el concepto de experiencia como un aspecto reconocido y reelaborado en el oficio docente basado en la relación y en la práctica de la enseñanza, la cual puede ser inclusiva al requerir de nuestra presencia, donde se promueve la atención a lo singular y lo común (López Carretero, 2010) y que responde a las características propias del alumnado. Por otra parte, la FID implica abrir o modificar un camino formativo en cuanto a la relación con los demás individuos y con el mundo.

Entendemos la FID como el proceso que viven quienes optan por la profesión docente para ser agentes socializadores y educativos que miran la escuela teórica y prácticamente (Hermida Vázquez et al., 2015). Reconocemos la responsabilidad que recae sobre la formación de los futuros cuerpos docentes, donde juega un papel importante la relación escuela y universidad en la FID, sobre todo en lo que respecta a la responsabilidad.

Desde el ámbito educativo se trabajan diversos aspectos para vivir en un contexto inclusivo que trascienda el poder del discurso para transformarse en un hacer, es decir, en acciones que incluyan dinámicas de compromiso con aquellos espacios y relaciones más inclusivas (Angenscheidt Bidegain y Navarrete Antola, 2017; San Martín et al., 2017). Con esto, expresamos el valor de nuestro estudio, ya que una mirada inclusiva supone forjar un frente común por el bienestar social (Pérez Santalla, 2017). El maestro y la maestra en formación pueden, a través de su acompañamiento, favorecer la inserción escolar, guiado por el deseo de que cada una de las criaturas encuentren un lugar propicio para su desarrollo (Márquez Valdés et al., 2017).

Crear posibilidades en la FID que partan de las incertidumbres y complejidades pone en evidencia los saberes que se vivifican y renuevan constantemente en los procesos creativos generados en el aula. Partiendo de la idea de que el saber del aula no es el ya dado, creemos que es fundamental prepararse para el oficio docente abriendo preguntas y poniendo en relación 
http://doi.org/10.15359/ree.25-3.8

saberes personales, tradiciones pedagógicas y disciplinares para repensar (Blanco García et al., 2015) y avanzar hacia saberes que interrogan, con los que se pueda conversar y abrir nuevas posibilidades y horizontes desde los que vivir e imaginarse como docentes que benefician contextos inclusivos. La clave formativa puede estar en cómo abrir y recoger experiencias para poner palabras y facilitar un proceso de pensamiento que desvele sentidos educativos.

\section{Metodología}

En este estudio se utilizaron relatos de experiencias (audiovisuales y escritos), lecturas y conversaciones, entre otros, para analizar y reflexionar de qué manera estas diferentes formas invitaban a abrir nuevos interrogantes e inquietudes que lleven a los sujetos participantes a pensarse como futuro personal docente dentro de un contexto social y educativo inclusivo (Alliaud y Suárez, 2011; Clandinin, 2013; Contreras Domingo, 2013; van Manen, 2003). Por lo tanto, vemos de qué manera la formación inicial puede abrirse a un proceso experiencial y narrativo que facilite la conexión con la escuela inclusiva.

En el proceso para analizar la experiencia educativa se realizó el siguiente recorrido metodológico, adaptado a partir de la indagación narrativa propuesta por Clandinin y Connelly (2000):

- Primera fase: elaboración de los textos de campo. Estos corresponden a las anotaciones de las observaciones de clase, de las conversaciones y los escritos del estudiantado. Estos textos iniciales además de contener los sucesos ocurridos también incluyen algunas reflexiones e interpretaciones de las investigadoras. En la indagación narrativa los datos son los textos de campo que son escritos como composiciones narrativas (Caine et al., 2013).

- Segunda fase: los textos de campo son analizados buscando hilos de sentido que pudieran ser valiosos para conformar los textos de investigación intermedios y que tuvieran relación con los objetivos del estudio. En esta fase se incluyen nuevas reflexiones e interpretaciones a las escenas, experiencias o relatos seleccionados. Estos textos pueden volver a ser usados como fuente de reflexión en el campo (Clandinin y Huber, 2010).

- Tercera fase: los textos intermedios fueron compartidos con la profesora que impartía la asignatura, para incluir nuevas reflexiones e interpretaciones, otorgándole renovadas miradas a los relatos.

- Cuarta fase: elaboración de textos de investigación final que vinculan los relatos con referentes teóricos y nuevas reflexiones e interpretaciones de la experiencia que se exponen en los apartados: resultados, análisis, discusión y conclusiones. 
http://doi.org/10.15359/ree.25-3.8

http://www.una.ac.cr/educare

educare@una.ac.cr

\section{Enfoque}

El presente estudio fue realizado desde una metodología cualitativa, situada en un enfoque de la indagación narrativa (IN), una perspectiva que le da valor a las vivencias a través de historias que nos contamos acerca de quiénes somos y que ahonda en la forma en que experimentamos el mundo desde un sentido subjetivo del quehacer humano (Clandinin, 2013; Clandinin y Connelly, 2000). En la investigación estuvimos atentas a las situaciones que se vivieron en el aula con el estudiantado participante, ya que sus historias y experiencias de vida eran consideradas fundamentales en el desarrollo de las clases y, por tanto, en el análisis que expondremos.

Desde un punto de vista teórico, las experiencias estudiadas son concebidas como un proceso continuo, basado en la idea de que estas tienen como base situaciones anteriores $y, a$ la vez, una proyección futura experiencial (Clandinin y Connelly, 2000; Moriña, 2017). Partimos de la base del sentido vivencial, personal, subjetivo e inesperado de la experiencia que cobra sentido cuando las personas nos narramos (Contreras Domingo y Pérez de Lara, 2010). Como apuntan Martín-Alonso et al. (2019), una pregunta metodológica fundamental es: ¿cómo orientarse a la experiencia del alumnado? Para lograrlo, una opción podría ser el desarrollo de una investigación que proponga una invitación al alumnado a compartir y describir sus vivencias a través de diferentes dinámicas en el aula.

La IN posee una naturaleza ontológica, ya que no es solo una metodología, sino también una manera de construir realidad (Bruner, 1991) donde las investigadoras y participantes están dentro de una relación dialógica y dialéctica (Clandinin, 2013) situándose en medio de vivir, contar, revivir y recontar las historias de las experiencias (Clandinin y Connelly, 2000).

La narrativa que se comparte en este estudio es un medio de acción formativa sobre la profesora, las investigadoras y también del posible lector (Barrios Tao, 2018) quien podrá realizar sus propias interpretaciones y reflexiones.

\section{Participantes}

La investigación se desarrolló en la asignatura Teoría y práctica de la escuela inclusiva en segundo año de la carrera de Educación Infantil donde había 43 estudiantes con edades entre 18 a 23 años, 39 mujeres y 4 hombres y tuvo una duración de un semestre. Se seleccionó esta asignatura por estar impartida por una de las investigadoras que incorpora el saber de la experiencia y la narrativa en sus clases.

\section{Técnicas de recolección}

Para el registro de textos de campo, que corresponden a los datos iniciales, hicimos un seguimiento de la asignatura durante un semestre, específicamente asistimos al aula, realizamos conversaciones con la maestra que impartía las clases y analizamos los trabajos escritos en 
http://doi.org/10.15359/ree.25-3.8

primera persona del estudiantado. Estos consistían en reflexiones iniciales y finales respecto a lo que significó para cada estudiante realizar la asignatura de Teoría y práctica de la escuela inclusiva desde una perspectiva que valoraba el saber y la experiencia personal que aportaba cada quien. A partir de ello reflexionamos sobre lo que dio de sí el repensar los procesos de formación docente, prestando atención a sus posibilidades y dificultades.

\section{Resultados. Lo que dio de sí}

\section{La experiencia vivida en el aula}

Al pensar en cómo llevar a cabo la asignatura, surgieron preguntas que guiaron el proceso, las cuales presentamos a continuación: ¿De qué forma podemos introducir y acercar la escuela inclusiva a los futuros maestros y maestras? ¿Cómo potenciar prácticas y saberes en el personal docente para que sea activo diseñador de entornos educativos inclusivos? ¿Cómo apoyar a estos grupos en formación para que, en el futuro, den respuesta al contexto inclusivo que, como comunidad educativa anhelamos? De ahí nació el objetivo de la propuesta para la asignatura, el cual es repensar la formación inicial para entrar en diálogo con prácticas que permitan acercarnos a una escuela inclusiva, relevando el saber de la experiencia del propio alumnado.

Respecto al quehacer durante la asignatura, además de mirar ejemplos y pensar cómo se va construyendo en comunidad una escuela inclusiva, se puso énfasis en el sentido propio (en la historia, perspectivas, relatos y aportación de cada persona) y en cómo quienes se están formando para ser educadores y educadoras han vivido experiencias de inclusión y exclusión a lo largo de su camino escolar, en la universidad y también en su contexto social y personal. Uno de los ejes de la asignatura es el autoconocimiento, pues explorar en nuestro interior es un requisito importante en el oficio educativo. Por eso, una de las prácticas que se propuso fue explorar conjuntamente cómo cada uno se veía a sí mismo, lo cual está estrechamente vinculado con las relaciones significativas con el mundo.

A partir de ciertas situaciones que se fueron proponiendo en las clases, el estudiantado reflexionó sobre la necesidad de crear espacios de relación en las aulas. También en cómo se viven -desde las miradas culturales y personales- las diferencias de ser hombre o mujer, planteándose las preguntas: ¿Cómo vivimos esas diferencias y cómo articulamos deseos y sentimientos? Desde ahí, se propuso reflexionar sobre cómo cada persona se sitúa en las relaciones con los otros seres. De un mismo hecho se pueden tener diferentes miradas aportadas por cada estudiante, ya que las relaciones humanas son subjetivas. A partir de estos planteamientos se fueron entretejiendo reflexiones en las clases.

En definitiva, se llevó a cabo una propuesta formativa que permitió al estudiantado explorarse dentro de sus propias historias y en sus saberes. Asimismo, se trabajó desde la 
http://doi.org/10.15359/ree.25-3.8

http://www.una.ac.cr/educare

educare@una.ac.cr

sensibilidad de las experiencias presentes, dentro y fuera del aula de formación, como fuente de saberes educativos.

Desde la idea de conectar con los saberes propios del estudiantado, algunas de las propuestas desarrolladas en clases fueron:

- La experiencia autobiográfica del estudiantado que emerge de sus vidas, de sus historias, de sus vivencias educativas escolares y su propia experiencia de formación en el aula universitaria.

- Las experiencias docentes susceptibles dellevar al aula universitaria: ejemplos de escuelas donde se estén llevando a cabo proyectos inclusivos, relatos elaborados por docentes; otros textos narrativos de tipo testimonial; películas documentales.

Para favorecer esta articulación entre experiencia, saber y sentidos educativos que se abren, utilizamos diferentes lenguajes narrativos que favorecieron la implicación en primera persona, pensando en relación con lo que le pasa al ser humano. En otras palabras, se puso especial cuidado tanto a las fuentes que se utilizaron (imágenes, relatos, películas); como a las propuestas que se le pidió al estudiantado para que materializaran sus experiencias y las pudieran expresar. En definitiva, utilizaron expresiones narrativas como: fotografías, cómics, pequeños guiones audiovisuales, relatos escritos y textos teóricos con los cuales conversar, pensar y entrelazar con la experiencia. De sus escritos seleccionamos algunos que aludieron a los aspectos que consideramos en esta investigación, por lo cual, pondremos extractos de sus palabras.

Desde el primer encuentro, la profesora pone en movimiento un aspecto que ella considera esencial; estar en cuerpo presente dando importancia a los espacios del aula para facilitar el encuentro y la interacción entre las personas. Las propuestas iniciales pretendían abrir el espacio para que el alumnado pudiese relatarse desde sí, expresando el sentir y saber que traía respecto a la escuela inclusiva. Allí se evidenció un discurso inicial políticamente correcto, ya que se tendía al buenísimo, expresión acuñada por la profesora que aludía a las buenas intenciones, que poco hablaban de la acción y del rol docente. Uno de los objetivos de la profesora de la asignatura era desplazar ese discurso para que en el aula hubiera un punto de inicio experiencial basándose en la historia de cada estudiante.

En las siguientes sesiones el estudiantado fue relatando y compartiendo vivencias propias en cuanto a la inclusión o exclusión en su etapa escolar, lo que permitió que algunos revivieran aspectos poco reflexionados con anterioridad. También hubo espacio para hablar de experiencias de otros.

A continuación, presentamos algunos extractos de lo que expresó el estudiantado en el trabajo final, que consistía en reflexiones escritas respecto a la experiencia vivida en el aula a partir de la propuesta didáctica: 
http://doi.org/10.15359/ree.25-3.8

Estudiante 18: Antes de esta asignatura, quizás, no le daba tanta importancia al hecho de que existe una singularidad en cada ser y si me hubieran preguntado si he vivido una situación de exclusión hubiera respondido con un no rotundo (el pensamiento general es: no tengo ninguna discapacidad física ni psíquica visible, por lo tanto, nunca he vivido una situación de exclusión). Pero creer eso es un error.

Estudiante 27: Pude reflexionar y darme cuenta de cosas que pasaba por alto. Las clases ayudaron a conocernos nosotras mismas y a pensar más en los demás y ponernos en su piel. Compartir reflexiones ayudó a conocer otras experiencias.

Estudiante 43: ...la clase se caracterizaba por ser dinámica, donde tenía más peso la práctica que la teoría y eso me permitió aprender y construir significados compartido a partir de la experiencia de nuestras compañeras, de la maestra y la mía propia. Una perspectiva nueva que me ha hecho reflexionar.

A partir del hecho de compartir, del ir y venir de ese dentro-fuera del sentido personal y colectivo que se fue articulando en la clase, se fue construyendo una visión de la escuela inclusiva dándole valor a las diferencias, desplazando poco a poco el discurso buenista inicial y acercándonos a acoger la búsqueda de opciones, de reconocer la diversidad y de abrirse a la incertidumbre que acompaña el oficio docente. Sobre esto algunos o algunas estudiantes dijeron:

Estudiante 16: La clase invita a la reflexión y plantearnos lo que hacemos y decimos hacer, a la evaluación de nuestros actos y pensamientos.

Estudiante 8: Pensamos en las diferencias como una posibilidad de crecimiento individual o grupal. Aprender a vivir la diversidad... en la escuela inclusiva entendida como aquella que se preocupa por cada uno de los estudiantes.

Al entrelazar las experiencias propias y compartidas con las lecturas de la asignatura se fueron dando pasos para narrarse en primera persona, escuchando de manera atenta y activa, y cultivando el intercambio. Al ir leyendo sus trabajos, pudimos ver que fueron surgiendo, en la mayoría, el desplazamiento inicial de la búsqueda de recetas estancadas para aplicarlas en el aula, hacia el reconocimiento del saber incierto que muchas veces queda fuera del diagnóstico práctico. Sobre esto, una estudiante señaló:

Estudiante 13: Aprendí que no hay sitio donde esté escrito el cómo afrontar situaciones con personas con discapacidad. Somos diferentes y únicos y, por lo tanto, hay diversidad de actuaciones frente a la diferencia. 
http://doi.org/10.15359/ree.25-3.8

http://www.una.ac.cr/educare

educare@una.ac.cr

Los relatos que escribió el estudiantado al inicio de la asignatura fueron anónimos, lo que permitió a muchos ir generando confianza y espacio para iniciar un recorrido narrativo, pero para otra parte implicó un dejarse estar y alejarse de la relación educativa que se esperaba. Por esta razón, la profesora sugirió elaborar relatos en primera persona, para que fueran tomando conciencia del espacio de relación requerido para crear entre todo el grupo. La intención era favorecer el acompañamiento y la contención a los que no se estaban sintiendo cómodos con esta manera de estar en el aula. Sin embargo, surgieron tensiones que expresamos a continuación:

Estudiante 39:... se requiere participación para lograr mayor interiorización y entender mejor, pero si no hay participación de todos se vuelve monótono y baja la motivación.

Estudiante 37: ... tenía el objetivo de captar la atención de los alumnos a través de la participación para ampliar conocimientos y profundizar. No lo aproveché debidamente porque no estaba acostumbrado a esta metodología.

Estudiante 15: Nos costó adaptarnos porque no nos conocíamos, no había confianza para hablar entre nosotros.

Estudiante 11:... no aproveché [la asignatura] como debería, no todos participamos, quizás fue porque no estábamos acostumbrados a considerar las experiencias propias en la universidad.

Los trabajos presentados y las vivencias de las clases fueron moviendo y articulando los saberes de todo el grupo: la maestra pudo ir entretejiendo nuevas formas de establecer relaciones educativas y el estudiantado no solo se abrió a ver los proyectos de escuela inclusiva diferente, sino también a un ser docente, como alguien que debe estar en constante escucha y cuestionamiento para atender lo incierto, conectando con lo particular de la vida en el aula, dialogando con los textos, con las ideas y con las experiencias:

Estudiante 25: Podíamos expresar nuestras opiniones, pensamientos y dudas en los debates. Por medio de esto, comprender de manera práctica los conceptos teóricos y hacer un gran trabajo reflexivo cambiando mi visión de la inclusión.

Estudiante 18: El ver historias explicadas en primera persona fue de gran ayuda para entender los conceptos trabajados en clases.

Estudiante 28: Aprendí de una manera diferente, donde las clases dependían de nosotros y de nuestras opiniones y aportaciones. Debíamos construir los aprendizajes (constructivismo) fue un aprendizaje colectivo basado en la reflexión y el pensamiento crítico. 
http://doi.org/10.15359/ree.25-3.8

La libertad para poder ser parte de la clase expresando sus sentimientos y opiniones es algo que se repite en sus relatos, como lo expresa la estudiante identificada con el número 28. La mayoría lo valora como algo positivo, además de sentir que sus ideas eran escuchadas y consideradas para poder ir perfilando los saberes de la asignatura.

Para acabar la recopilación de relatos, compartimos la reflexión de la profesora:

Docente de la asignatura: Los relatos son una mediación para entrelazar las experiencias y acercarse desde ahí a un pensamiento educativo original en la interacción y articulación constante entre ser y saber. Una mediación para inspirar la experiencia de hacer algo propio con el saber que les permita conectar con el sentido de las prácticas educativas. Trabajar de esta forma permite aprender a narrar y narrarse partiendo de sí, es decir, para que la conversación y la escritura sean modos de interiorizar y extraer pensamiento de la experiencia: para aprender a escuchar y escucharse de otro modo y entender los sentidos que mueven las prácticas educativas. Lo que hemos hecho es hablar y escribir en primera persona. Entender el hablar desde sí y no de mí, como un modo de subjetivar las propias experiencias de los estudiantes a partir de los relatos propios y los de otros.

\section{Análisis y discusión}

En los relatos se puede ver una apertura a la reflexión donde las experiencias y las prácticas vividas en primera persona fueron las protagonistas, donde existe una libertad para expresar y escuchar vivencias, opiniones y sentimientos de quienes están presentes en la relación educativa. Así se pone en acción real aquello que se ve a nivel teórico y que respalda la idea de inclusión.

Al hacer un análisis de las reflexiones finales del alumnado, surgió el tema de la falta de espacios en la universidad para colocarse en primera persona. La razón indicada por la profesora es que en los programas y planes de las asignaturas se tiende a tratar mucha teoría o modelos conceptuales y, escasamente, se incorpora la experiencia. Pero en el desarrollo de la propuesta didáctica que seguimos, hubo una especial dedicación a las reflexiones vinculadas a las historias de vida del alumnado. En ese quehacer nos encontramos con algunas cuestiones relacionadas con el itinerario escolar previo que, en algunos casos, era inclusivo y en otros no. Un aspecto común fue la falta de confianza en que su experiencia tuviese lugar en la academia. Como señalamos más arriba, en las aulas universitarias, específicamente en las asignaturas destinadas a la formación, es fundamental reconocer la importancia de la diversidad en las escuelas (Huguet i Comelles, 2006) y eso se puede lograr con dinámicas como la llevada a cabo en la asignatura que presentamos.

Otro aspecto que tomamos en cuenta fue la necesidad de respuestas disponibles en las situaciones prácticas y las resistencias para ir elaborando caminos desde las propias vivencias. Según los registros recopilados, suele ser mucho más fácil hablar desde la teoría sin incluir el 
http://doi.org/10.15359/ree.25-3.8

http://www.una.ac.cr/educare

educare@una.ac.cr

propio pensamiento en el desarrollo de las clases y en los productos que se van elaborando a lo largo de la asignatura. El estudiantado mostró ciertos inconvenientes a la hora de utilizar recursos expresivos de escritura narrativa, en los que pudieran reflejar la complejidad de las situaciones educativas o con los que poner en juego las dimensiones subjetivas de la experiencia. Implementar prácticas reflexivas, como narrarse desde sí en la universidad, nos permite pensar que contar con saberes y experiencias propias es fundamental para visualizar dimensiones y prácticas educativas que proyecten escuelas inclusivas desde lo real y, así encaminarnos a lo que significa crear espacios donde se valoren las diferencias y las cualidades de cada persona. En suma, el integrar la experiencia en la formación del profesorado es una práctica que puede ir generando cambios.

A las tensiones expresadas anteriormente, que surgen en algunos momentos vividos en el aula, les hemos prestado atención, ya que consideramos que no es fácil el desplazamiento de su lugar de alumnos para mirarse como futuros educadores que elaboran pensamiento propio. Caminar por esta senda es compleja porque emergen de la sensibilidad y atención en las relaciones y comunicación entre el alumnado y el profesorado.

\section{Conclusiones}

El actual itinerario formativo universitario plantea la necesidad de posicionarnos en un nuevo marco, uno que incluya prioridades orientadas a un carácter actualizado e innovador respecto a la formación general de maestros y maestras (Blanco García et al., 2015; González Olivares y Blanco García, 2015). Para el estudio, nos centramos en observar y hacer un seguimiento a las estrategias didácticas en un aula universitaria. En ese contexto, partimos de la idea de indagar en la dimensión subjetiva y personal que caracteriza la experiencia, y que nos exige una forma de estar en primera persona y en relación con el otro ser (López Carretero, 2010) pensando en escuelas inclusivas que respeten la diversidad.

Respondiendo al objetivo de reflexionar sobre la incorporación del saber de la experiencia en la formación inicial del profesorado, usando como fuente el relato de una profesora que implementa una propuesta didáctica inclusiva, en el apartado de resultados se pudo constatar que, el aspecto autobiográfico a partir del relato oral (conversaciones en clases) y de la escritura (trabajos reflexivos individuales), el estudiantado fue rescatando hechos y vivencias que habían tenido en su etapa escolar para pensar en la inclusión o exclusión en primera persona. También pudieron considerar la manera en que vivían la formación en la universidad, para analizar si en ella había dinámicas inclusivas donde se valore al ser humano con sus diferencias y opiniones. En este sentido, lo que le interesaba a la profesora era potenciar un espacio de aprendizaje en la universidad que en sí mismo fuera una vivencia de inclusión.

Por otro lado, al poder contar con las miradas de otras personas, a través de experiencias de distintos agentes vinculados al mundo educativo, pudieron reflexionar sobre lo que implica 
http://doi.org/10.15359/ree.25-3.8

un proyecto inclusivo y al contar con algunos ejemplos de escuelas hubo un acercamiento a los complejos procesos que se viven para lograr la inclusión en diferentes contextos.

A lo largo de la asignatura Teoría y práctica de la escuela inclusiva se fue trabajando desde una mirada que valoraba el saber de la experiencia para tratar temas como: la diferencia, la diversidad, la alteridad y la subjetividad, además del tema central que era la escuela inclusiva. Lo que nos lleva a volver a preguntarnos: ¿qué saberes docentes reclama la formación inicial para tratar temas relacionados a la escuela inclusiva? Al respecto, esta perspectiva permitió ir repensando la formación que dé cabida a crear, participary vivir proyectos educativos inclusivos, que se acerque a los saberes encarnados que podrían desarrollarse para ello.

Conesta investigación abogamos por un sentido dela educación como un lugar compartido que cuestiona aspectos de lo que significa la inclusión, partiendo de las historias de quienes están en proceso de formación. Entre las principales reflexiones está el valor de tener como punto de partida la propia experiencia, con ella será más fácil comprender, desde la práctica, lo que exige la escuela inclusiva, porque ese proyecto demanda una filosofía que no excluye el sentido de lo propio; al contrario, sí se incorpora la experiencia, el nivel de sensibilización y de proyección como futuros grupos docentes podría ser mayor.

En este sentido, la experiencia relatada permitió tomar conciencia sobre la importancia de ser partícipes en la creación de contextos inclusivos. Es esa mirada la que nos permite replantear nuestra perspectiva a la hora de hablar de educación inclusiva.

En el marco teórico señalábamos que en las aulas universitarias se tiende a hacer un análisis crítico de la función de la escuela (Huguet i Comelles, 2006), pero desde un sentido lejano, sin ponerse en primera persona en las dinámicas de creación o cambio que se pueda producir en ella. Por lo tanto, hacer un recorrido por la historia personal del estudiantado puede ayudar a ver desde otra perspectiva la función de la escuela, ya que la mayoría hemos vivido experiencias escolares y, desde allí, se pueden ampliar perspectivas de cambios que aporten en la FID.

Algunas preguntas que surgieron fueron: ¿qué prácticas se llevan a cabo en las aulas universitarias para pensar en la diversidad de las escuelas?, ¿se reflexiona sobre el lugar propio que se ocupa mientras se viven procesos de formación? Al realizar este estudio pudimos constatar que las dinámicas puestas en práctica, en esta aula en particular, promovían la escucha y la atención; elementos que podrían ser claves para crear espacios inclusivos. En ese sentido, las clases que observamos eran un ejemplo del quehacer para pensarse como futuros y futuras docentes que gestionan espacios inclusivos en las escuelas. Además, el planteamiento de una escuela inclusiva no iba tan solo por una vía teórica con la habitual pregunta: ¿qué se entiende por escuela inclusiva? El sentido de la propuesta curricular que plantea la profesora era el de responder a esta interrogante incorporando la percepción personal y las vivencias de cada sujeto, con el objetivo de proyectar una idea de escuela que se fundamenta en la creación común de proyectos que miran a cada persona desde el valor de la diferencia y la alteridad. 
http://doi.org/10.15359/ree.25-3.8

http://www.una.ac.cr/educare

educare@una.ac.cr

Por otra parte, la docente le daba mucha importancia a dar lugar y escuchar la voz propia del estudiantado para cultivar la relación con el saber desde las preguntas e inquietudes que pueda tener cada persona. También le daba valor a la experiencia de estar en la universidad, por ser una oportunidad que ayuda a descubrir nuevos sentidos de lo educativo para imaginar posibilidades inclusivas en el mundo.

Nos llamó la atención que en las clases apareciera el concepto de libertad como un valor presente en la formación -y que no siempre se reconoce en la universidad-, donde cada quien va forjando sentidos que pueden transformar proyectos educativos y que definen la vía que toma para su aprendizaje, partiendo de la mirada a la propia persona y del tipo de interrelaciones que se generan en espacios como las aulas universitarias o como las escuelas.

En relación con las dificultades que surgieron al incorporar el saber de la experiencia, se pudieron percibir algunas tensiones que fueron comentadas con la profesora que impartía las clases: 1) el alumnado había vivido un itinerario escolar y universitario previo con pocas libertades, lo que implicó un dejarse estar, para una parte, frente a una propuesta con mayor libertar; 2) un grupo demostró una falta de confianza a la hora de expresar vivencias propias en los espacios que se le brindaba en la clase; 3 ) había una necesidad de respuestas estandarizadas que identificamos al principio de la asignatura, ya que los estudiantes y las estudiantes expresaron que necesitaban estrategias para llevar a cabo proyectos inclusivos, como recetas factibles de aplicar en las escuelas y, por último, 4) dificultades iniciales para narrarse desde sí a través de la escritura y la oralidad.

Finalmente, cuando nos preguntamos qué saberes reclama la formación inicial para tratar temas relacionados a la escuela inclusiva, pensamos que el oficio docente nos exige buscar formas que permitan articular la experiencia con los saberes desde una mirada propia. Un saber pedagógico que se denomina saber de la experiencia (Clandinin, 2013; Contreras Domingo, 2013) o como lo llama van Manen (2003): conocimientos páticos. Nombrar el saber de la experiencia nos permite hacer hincapié en ese carácter encarnado, integrado, existencial y personal; un saber que va más allá de lo cognitivo o de la posesión de conocimientos y que, a la vez, integra disposiciones y relaciones que demandan una actitud abierta a la escucha (López Carretero, 2010), al respeto y a la sensibilidad de lo que se vive en contextos en los que todo el ser está involucrado para crear proyectos inclusivos.

\section{Referencias}

Ainscow, M. (2001). Desarrollo de escuelas inclusivas. Ideas, propuestas y experiencias para mejorar las instituciones escolares. Narcea.

Ainscow, M., Dyson, A., Goldrick, S. y West, M. (2012). Making schools effective for all: Rethinking the task. School Leadership \& Management, 32(3), 197-213. https://doi.org/10.1080/13632 $\underline{434.2012 .669648}$ 
http://doi.org/10.15359/ree.25-3.8

http://www.una.ac.cr/educare educare@una.ac.cr

Alliaud, A. y Suárez, D. H. (Coords.) (2011). El saber de la experiencia. Narrativa, investigación y formación docente. CLACSO-UBA.

Angenscheidt Bidegain, L. y Navarrete Antola, I. (2017). Actitudes de los docentes acerca de la educación inclusiva. Ciencias Psicológicas, 11(2), 233-243. https://doi.org/10.22235/ cp.v11i2.1500

Arbiol i González, C., Arnaus i Morral, R., Blanco García, N., Contreras Domingo, J., Gabbardini, P., López Carretero, A., Molina Galvañ, M. D., Serra. A. N., Orozco Martínez, S. y Ventura Robira, M. (2016). Tensiones fructíferas: Explorando el saber pedagógico en la formación del profesorado. Una mirada desde la experiencia. Octaedro.

Arostegui Barandica, I. y Darretxe Urrutxi, L. (2016). Estrategias metodológicas activas en la asignatura de "Bases de la Escuela Inclusiva" de la E. U. de Magisterio de Bilbao. REDU. Revista Docencia Universitaria, 14(2), 315-340. https://doi.org/10.4995/redu.2016.5986

Barrios Tao, H. (2018). Racionalidad narrativa en procesos de investigación-formación en educación. Revista Colombiana de Ciencias Sociales, 9(2), 478-502. https://www.funlam. edu.co/revistas/index.php/RCCS/article/view/2523/pdf

Blanco García, N., Molina Galvañ, M. D. y López Carretero, A. (2015). Aprender de la escuela para dar vida a la universidad. Revista Interuniversitaria de Formación del Profesorado, 82(29.1), 61-76. https://dialnet.unirioja.es/servlet/articulo?codigo $=5132096$

Bruner, J. (1991). The narrative construction of reality. Critical Inquiry, 18(1), 1-21.

Caine, V., Estefan, A.y Clandinin, D. J. (2013). A return to methodological commitment: Reflections on Narrative Inquiry. Scandinavian Journal of Educational Research, 57(6), 574-586. https:// doi.org/10.1080/00313831.2013.798833

Caine, V., Estefan, A. y Clandinin, D. J. (2019). Narrative nquiry. En P. Atkinson, S. Delamont, A. Cernat, J. W. Sakshaug y R. A. Williams (eds.), SAGE Research Methods Foundations (pp. 1-23). https://doi.org/10.4135/9781526421036

Clandinin, D. J. (2013). Engaging in narrative inquiry. Left Coast Press.

Clandinin, D. J. y Connelly, F. M. (2000). Narrative inquiry: Experience and story in qualitative research. Jossey-Bass.

Clandinin, D. J. y Huber, J. (2010). Narrative inquiry. En B. McGaw, E. Baker y P. P. Paterson (Eds.), International Encyclopedia of Education (3. ${ }^{a}$ ed., pp. 436-441). https://doi.org/10.1016/ B978-0-08-044894-7.01387-7

Contreras Domingo, J. (2013). El saber de la experiencia en la formación inicial del profesorado. Revista Interuniversitaria de Formación del Profesorado, 78(27,3), 125-136. https://dialnet. unirioja.es/servlet/articulo?codigo $=4688508$ 
http://doi.org/10.15359/ree.25-3.8

http://www.una.ac.cr/educare

educare@una.ac.cr

Contreras Domingo, J.y Pérez de Lara, N. (Comps.). (2010). Investigar la experiencia educativa. Morata.

De la Rosa Moreno, L. (2017). Objetos convertidos en sujetos: Encuentros con voces excluidas dentro de una asignatura sobre inclusión educativa. Revista Electrónica Interuniversitaria de Formación del Profesorado, 20(3), 209-223. https://doi.org/10.6018/reifop.20.3.260111

Duk, C., Cisternas, T. y Ramos, L. (2019). Formación docente desde un enfoque inclusivo. A 25 años de la declaración de Salamanca, nuevos y viejos desafíos. Revista Latinoamericana de Educación Inclusiva, 13(2), 91-109. https://dx.doi.org/10.4067/S0718-73782019000200091

Durán Gisbert, D. y Giné Giné, C. (2017). La formación del profesorado para la educación inclusiva: Un proceso de desarrollo profesional y de mejora de los centros para atender la diversidad. http://www.repositoriocdpd.net:8080/handle/123456789/1913

García García, M. y Benitez Gavira, R. (2014). Comprometiéndonos con "nuestra escuela": Un proyecto de aprendizaje-servicio para una formación del profesorado inclusiva. Revista nacionaleinternacionaldeeducacióninclusiva,7(2),69-83.https://revistaeducacioninclusiva. es/index.php/REl/article/view/147/141

González Olivares, Á. L. y Blanco García, M. (2015). Formación del profesorado de educación inclusiva: Reto docente de la educación especial. Opción, 31(3), 582-604. https://www. redalyc.org/articulo.oa?id=31045567030

Hermida Vázquez, N. A., López Rodríguez del Rey, M. M. y Díaz Vera, C. E. (2015). Las relaciones entre la universidad y la escuela: Su contribución al aprendizaje en la formación del profesorado. Revista Universidad y Sociedad, 7(3), 32-39. http://scielo.sld.cu/scielo. php?script=sci arttext\&pid=\$2218-36202015000300006\&lng=es\&tlng=es

Huguet i Comelles, T. (2006). Aprendre junts a l'aula. Una proposta inclusiva. Graó.

López Carretero, A. (2010). Un movimiento interior de vida. En J. Contreras y N. Pérez de Lara (Comps.), Investigar la experiencia educativa (pp. 211-224). Morata.

Marcelo,C.,Yot, C., Mayor,C.,Sánchez Moreno, M.y Murillo,P.(2014).Las actividades deaprendizaje en la enseñanza universitaria: ¿Hacia un aprendizaje autónomo de los alumnos? Revista de Educación, (363), 334-359. https://doi.org/10.4438/1988-592X-RE-2012-363-191

Márquez Valdés, A. M., Acosta, Bandomo R. U. y García Ruiz, M. B. (2017). La formación didáctica inicial del maestro para la educación especial. Una mirada a la experiencia cubana. Revista Nacional e Internacional de Educación Inclusiva, 10(2), 31-41. https:// revistaeducacioninclusiva.es/index.php/REl/article/view/308

Martín-Alonso, D., Blanco García, N. y Sierra Nieto, J. E. (2019). El proceso de creación curricular en estudiantes de educación secundaria. Una indagación narrativa. Profesorado. Revista de Currículum y Formación de Profesorado, 23(2), 377-395. https://doi.org/10.30827/ profesorado.v23i2.9692 
http://doi.org/10.15359/ree.25-3.8

http://www.una.ac.cr/educare educare@una.ac.cr

Martínez Tornay, A. (2014). La formación inicial y permanente del profesorado para dar respuesta a la diversidad: Propuestas y retos para una educación inclusiva de calidad y de excelencia [Tesis doctoral]. Universidad Complutense de Madrid. https://eprints.ucm.es/id/eprint/27668/1/ T35492.pdf

Mendonça, F. L. de Rezende y Silva, D. N. H. (2015). A formação docente no contexto da inclusão: Para uma nova metodologia. Cuadernos de Pesquisa, 45(157), 508-526. https://doi. org/10.1590/198053143274

Moriña, A. (2017). Investigar con historias de vida. Metodología biográfica-narrativa. Narcea.

Moriña Díez, A. y Parrilla Latas, Á. (2006). Criterios para la formación permanente del profesorado en el marco de la educación inclusiva. Revista de Educación, (339), 517-539. http://www. educacionyfp.gob.es/dam/jcr:069c189a-9d96-4743-aed8-5fafc5a3444e/re33923-pdf.pdf

Pérez Santalla, I. (2017). Herramientas para la inclusión: De la educación a la sociedad. Revista Nacional e Internacional de Educación Inclusiva, 10(2), 13-30. https:// revistaeducacioninclusiva.es/index.php/REl/article/view/307

Porta, L., de Laurentis, C. y Aguirre, J. (2015). Indagación narrativa y formación del profesorado: Nuevas posibilidades de ruptura y construcción en la identidad docente. Praxis Educativa, 19(2), 43-49. https://cerac.unlpam.edu.ar/index.php/praxis/article/view/1039/972

Ramos Torres, S. C. y Galindo Rodríguez, D. F. (2018). La escuela en la universidad y la universidad en la escuela, dos mundos inherentes. En J. S. A. Perilla Granados (Comp.), Diseño curricular y transformación de contextos educativos desde experiencias concretas (pp. 301-329). Universidad Sergio Arboleda. https://repository.usergioarboleda.edu.co/ bitstream/handle/11232/1549/La\%20escuela\%20en\%20la\%20universidad\%20y\%20 la\%20universidad.pdf?sequence=1\&isAllowed $=y$

Rivera Iribarren, P. (2017). Educación inclusiva y formación inicial docente. Una experiencia en la región de Coquimbo, Chile [Tesis doctoral]. Universidad de Alcalá. https://ebuah.uah.es/ dspace/handle/10017/38236

Rojas Cely, R. y Quintero Peñaranda, O. A. (2013). Diversidad y educación inclusiva para la formación de docentes. Revista Hojas y Hablas, (10), 119-127. http://revistas.unimonserrate. edu.co:8080/hojasyhablas/article/view/17

San Martín, C., Villalobos, C., Muñoz, C. y Wyman, I. (2017). Formación inicial docente para la educación inclusiva. Análisis de tres programas chilenos de pedagogía en educación básica que incorporan la perspectiva de la educación inclusiva. Calidad en la educación, (46), 20-52. https://doi.org/10.4067/S0718-45652017000100020 
http://doi.org/10.15359/ree.25-3.8

http://www.una.ac.cr/educare

educare@una.ac.cr

Simón, C., Echeita, G. y Sandoval, M. (2018). Incorporating students' voices in the 'Lesson study' as a teacher-training and improvement strategy for inclusion. Culture and Education, 30(1), 205-225. https://doi.org/10.1080/11356405.2017.1416741

Van Manen, M. (2003). Investigación educativa y experiencia vivida. Idea Books.

Vélez-Calvo, X., Tárraga-Mínguez, R., Fernández-Andrés, M. I. y Sanz-Cervera, P. (2016). Formación inicial de maestros en educación inclusiva: Una comparación entre Ecuador y España. Revista de Educación Inclusiva, 9(3), 75-94. https://revistaeducacioninclusiva.es/index.php/ REI/article/view/254 